\title{
Computations of Flow over a Hump Model Using Higher Order Method with Turbulence Modeling
}

\author{
P.Balakumar* \\ NASA Langley Research Center, Hampton, VA 23681
}

\begin{abstract}
Turbulent separated flow over a two-dimensional hump is computed by solving the RANS equations with $k-\omega$ (SST) turbulence model for the baseline, steady suction and oscillatory blowing/suction flow control cases. The flow equations and the turbulent model equations are solved using a fifth-order accurate weighted essentially nonoscillatory (WENO) scheme for space discretization and a third order, total variation diminishing (TVD) Runge-Kutta scheme for time integration. Qualitatively the computed pressure distributions exhibit the same behavior as those observed in the experiments. The computed separation regions are much longer than those observed experimentally. However, the percentage reduction in the separation region in the steady suction case is closer to what was measured in the experiment. The computations did not predict the expected reduction in the separation length in the oscillatory case. The predicted turbulent quantities are two to three times smaller than the measured values pointing towards the deficiencies in the existing turbulent models when they are applied to strong steady/unsteady separated flows.
\end{abstract}

\section{Introduction}

Computing unsteady separated turbulent flows accurately and efficiently is currently a challenging problem in CFD research. Most of the computational codes at present use implicit second order methods with one or two equation turbulence models with multi grid convergence acceleration techniques. Higher order methods are successfully used in direct numerical simulation (DNS) of stability and transition of shear flows, of turbulent shear flows at low Reynolds numbers and in Large Eddy Simulations (LES) of shear flows. Time integrations are performed using fourth order Runge-Kutta type algorithms and space discretizations are performed using fourth or higher order central, upwind, compact or spectral methods with selected higher order filtering when necessary. High operating Reynolds numbers and complex configurations hinder applying these schemes to engineering calculations and in practice modeled Reynolds Averaged Navier-Stokes (RANS) equations are solved to obtain engineering quantities. The current status and the issues related to higher order methods are discussed in Ref. 1.

In the finite difference formulation, higher order spatial discretization requires larger stencil size and smooth grids. This makes simple extension of the higher order methods to complex geometries difficult. Block structured or overset grid systems with interpolation techniques near the interfaces are required to overcome these difficulties. Among the higher-order schemes, compact schemes ${ }^{2}$ and upwindbased schemes ${ }^{3}$ are pursued as viable algorithms to develop higher order codes. Compact schemes are global in character and in general have symmetric coefficients, hence have low dispersion and dissipation properties and provide high resolution with minimum number of grid points. However, due to the low dissipation, high frequency oscillations are generated and artificial damping or filtering are required to remove these instabilities. Another issue with these schemes is that across flow discontinuities and near under resolved large gradients they exhibit the classical Gibbs phenomena and the schemes have to be modified to upwind biased flux splitting type schemes and this may be difficult to implement in a general

* Aerospace Engineer, Flow Physics and Control Branch, NASA Langley Research center, MS 170, AIAA Member

1 of 16

American Institute of Aeronautics and Astronautics 2005-1270 
setting. Compact schemes have been applied to a range of problems ${ }^{4}, 5,6$. Higher order upwind based methods are essentially non-oscillatory (ENO) type methods that are designed to suppress the oscillations near discontinuities and larger gradient regions. They are robust and can be applied to a wide range of Mach number flows without any modifications. However due to the upwind biased stencil and flux splitting they are more dispersive and dissipative compared to compact schemes.

The objectives of this work are to apply a higher order accurate finite difference code to complex turbulent flows and to validate the computations against the experimental results. Three dimensional compressible unsteady Navier-Stokes equations are solved using a fifth order spatially accurate weighted essentially non-oscillatory (WENO) scheme and third-order accurate total variation diminishing (TVD) Runge-Kutta integration scheme. This is an upwind biased conservative scheme designed to suppress the oscillations near the discontinuities and large gradients. This method has been successfully applied to high Reynolds number laminar flows to investigate shock boundary layer interactions ${ }^{7}$, the stability and transition of supersonic boundary layers induced by roughness and acoustic waves ${ }^{8}$, and the screech tone generated in an imperfectly expanded supersonic jet ${ }^{9}$. Further development of this code is to extend this method to include turbulence modeling, and advanced modeling like DES and LES capabilities where low numerical dissipation is advantageous in discerning the effects of the sub-grid scale models from that introduced by numerical dissipation. In this paper, computations are performed for a turbulent flow over a two-dimensional hump model for the baseline, steady suction and oscillatory (suction/blowing) separation control cases by solving the RANS equations with $\mathrm{k}-\omega$ (SST) model using a higher-order finite difference code. A careful experiment has been performed for this configuration at NASA Langley Research center and the detailed experimental results are presented in the CFD validation workshop reports ${ }^{10}$ and in Ref. 11. The model and the experiments are similar to the work performed by Seifert and Pack $^{12}$.

\section{Experimental set up}

The experimental set up consists of a Glauret-Goldschmied type body mounted on a splitter plate. The chord length of the model is $c=16.53$ inches and the height is 2.11 inches. The leading and the trailing edges are smoothly flared with the splitter plate and the height from the splitter plate to the upper wind tunnel wall is 15.03 inches. The splitter plate is 0.5 inches thick and extends 76.18 inches upstream and 44.45 inches downstream from the leading and trailing edges of the model. Tripping is applied at the leading edge of the splitter plate to obtain a fully developed turbulent boundary layer over the model. Steady suction and unsteady zero mass flux synthetic jet controls are applied through the slot opening located between 10.81-10.88 inches from the leading edge of the model. The model has two end-plates at both sides of the hump. The distance between the endplates is approximately 23 inches. The experiments are performed for a wide range of flow parameters such as Reynolds number, Mach number, different steady suction rates, different unsteady frequencies, and unsteady flow rates. An elaborate data acquisition system including streamwise and spanwise surface pressure measurements, pitot tube and hot wire measurements, 2-D and 3-D PIV and LDV and skin friction measurements were used to gather very detailed steady and unsteady flow field data. The experiments showed that the flow field is nominally two-dimensional in the center part of the model. The computations are performed for a two-dimensional model.

\section{Computational set up}

The computational domain extends from $x / c=-10.0$ to 4.0 in the streamwise direction and extends from the splitter plate to the upper tunnel wall in the normal direction. The leading edge of the splitter plate is modeled as a super ellipse with 0.25 inches half-thickness and an aspect ratio of 2 . The 
leading edge of the splitter plate is located at $x / c=-5.9$ and the leading edge of the hump model is located at $x / c=0.0$. The length of the splitter plate is selected to match the measured velocity profiles at $x / c=$ 2.1. A schematic diagram of the computational model is shown in Fig. 1. The free-stream Mach number is 0.1 and the chord Reynolds number is 936,000 . In the steady suction case, the suction flow rate is 23 $\mathrm{cfm}$ through the suction slot across the span of 23 inches, and in the oscillatory case the frequency of actuation is $137 \mathrm{~Hz}$. and the maximum exit velocity is $27 \mathrm{~m} / \mathrm{s}$.

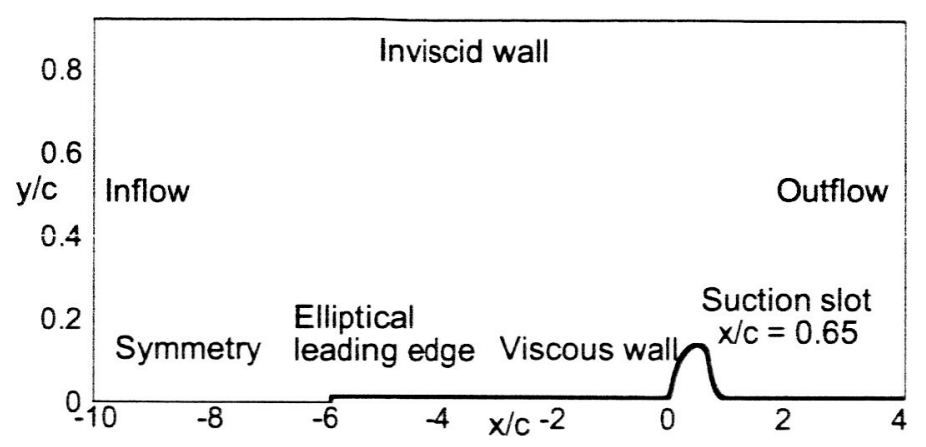

Figure 1. Schematic diagram of the hump model.

\section{Numerical Method}

The governing equations, the flow equations and the turbulent equations, are solved using the $5^{\text {th }}$ order accurate weighted essentially non-oscillatory (WENO) scheme for space discretization and using explicit third order total-variation-diminishing (TVD) Runge-Kutta scheme for time integration. The WENO and the TVD methods and the formulas are explained in Ref. 13 and the application of ENO method to N-S equations is given in Ref. 14. The solution method implemented in this computation is described in detail in Ref. 7. Standard $k$ - $\omega$ (SST) model is used and the equations and the model coefficients are described in Refs. 15-18. In the following presentation, $(x, y)$ are the Cartesian coordinates, $(u, v)$ are the velocity components, $\rho$ is the density, and $p$ is the pressure. $E$ is the total energy given by

$$
E=e+\frac{u^{2}+v^{2}}{2}, e=c_{v} T, p=\rho R T
$$

Here $e$ is the internal energy, $T$ is the temperature, $c_{v}$ is the specific heat at constant volume and $R$ is the gas constant. The viscosity $(\boldsymbol{\mu})$ is computed using Sutherland's law and the Prandtl number is taken as a constant value of 0.72 . For turbulent quantities $k$ is the turbulent kinetic energy, $\omega$ is the dissipation rate and $\mu_{T}$ is the turbulent eddy viscosity. In the following subscript ' $w$ ' denotes the values at the wall and the subscript ' $x$ ' denotes the free stream values.

A C-Type grid is used around the splitter plate and a rectangular grid is added upstream of the leading edge as shown in Fig. 2. The rectangular grid overlaps the $\mathrm{C}$ grid and $5^{\text {th }}$ order central interpolation is used to transfer the flow variables from one grid to the other at the boundaries. A grid size of $(651 * 151)$ is used around the splitter plate and the hump and a grid size of $(101 * 51)$ is used in the rectangular region. 


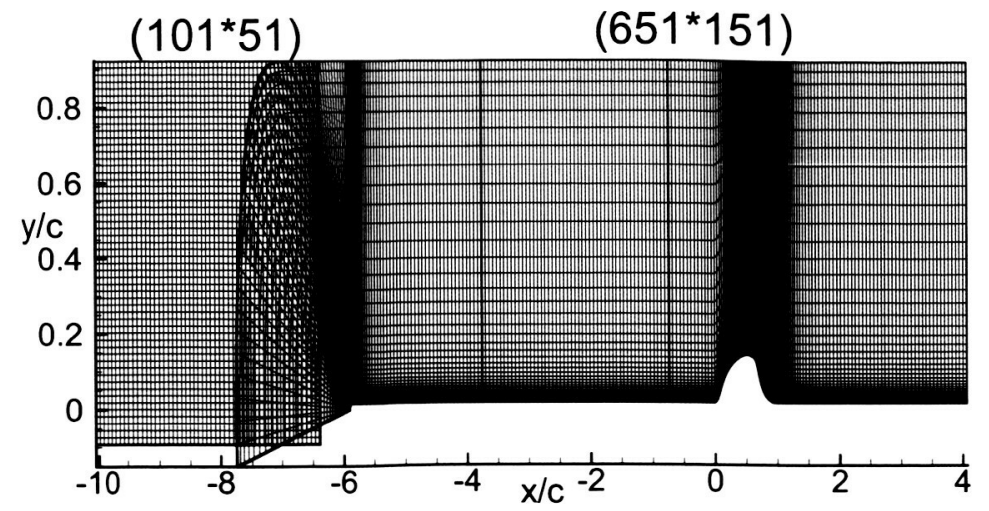

Figure 2: Overlapping grid near the leading edge of the splitter plate.

\section{A. Boundary conditions}

The following boundary conditions are implemented at different boundaries:

1. At the upper wall inviscid conditions are applied.

$$
\frac{\partial \rho}{\partial y}=\frac{\partial u}{\partial y}=\frac{\partial E}{\partial y}=v=0
$$

2. At the lower wall viscous conditions are used.

$$
\begin{aligned}
& u=v=0, \\
& T=T_{w}=T_{\text {freestream }},
\end{aligned}
$$

and $\rho$ is computed from the continuity equations.

3. From the leading edge of the splitter plate to the inflow boundary, symmetric conditions are used.

4. At the inflow boundary stagnation pressure, one Riemann variable and normal velocity $v=0$ are prescribed and the second Riemann variable is solved to obtain the other flow quantity.

5. At the outflow boundary the pressure is specified to obtain the required Mach number and characteristic-type boundary conditions are implemented similar to that described in Ref. 19 to obtain other flow variables.

6. In the suction case, boundary conditions are applied on the surface of the hump across the suction slot. The suction slot extends from $x / c=0.654$ to 0.658 and across the slot normal mass flow rate is specified to match the experimental value of $27.13 \mathrm{ft}^{3} /$ minute through the 23 inches span slot. A suction distribution of the form

$$
(\rho v)_{n}=f_{\max } \sin ^{2}\left(\frac{\pi\left(x-x_{\text {start }}\right)}{\left(x_{\text {end }}-x_{\text {start }}\right)}\right)
$$

is used to get the required mass flow rate. Here $f_{\max }$ is the maximum amplitude of the suction distribution selected to match the experimental total suction rate. Other forms have been tried and all of them yield the same results for a fixed total suction rate. The other flow quantities are obtained by extrapolation from inside the domain.

7. In the oscillatory control case, boundary conditions similar to the steady suction case are implemented. A blowing/suction distribution in the form

$$
(v)_{n}=v_{\max } \sin ^{2}\left(\frac{\pi\left(x-x_{\text {start }}\right)}{\left(x_{\text {end }}-x_{\text {start }}\right)}\right) \sin (\omega t)
$$

is prescribed for the velocity component normal to the slot. Here $v_{\max }$ is the maximum normal oscillating velocity selected to match the experimental value. During the blowing cycle the 
tangential velocity is obtained by assuming that the jet enters the flow domain at an angle of 30 degrees to the surface of the hump, and during the suction cycle the other flow quantities are obtained by extrapolation from inside the domain. The maximum normal velocity and the frequency of oscillations are selected to match the experimental conditions of maximum exit velocity of $27 \mathrm{~m} / \mathrm{s}$ and the oscillation frequency of $137 \mathrm{~Hz}$.

The following boundary conditions are implemented for the turbulent quantities at different boundaries.

1. At the inflow boundary, small values are prescribed for $k$ and $\mu_{T}$.

$$
\begin{aligned}
& \frac{k}{U_{\infty}^{2}}=10^{-7}, \\
& \frac{\mu_{T}}{\mu_{\infty}}=.009 .
\end{aligned}
$$

2. At the outflow boundary, $k$ and $\omega$ are solved for from the governing equations. Higher order extrapolation condition is also tried and it gives the same results.

3. At the lower viscous wall, $k=0$ condition is used and the exact boundary condition is derived for $\omega$ as described below.

4. In the steady suction and oscillatory control cases, across the suction slot, linear extrapolation is used to obtain the turbulent quantities on the surface.

Since the variable $\omega$ becomes singular near a viscous wall, in practice a large approximate value is prescribed at the wall. The turbulent model equations and the model coefficients are given in Ref. 18 .

$$
\omega_{\text {wall }}=\frac{60 \mu_{w}}{\rho_{w} \beta d_{w}^{2}},
$$

where $d_{w}$ is the distance to the first grid point from the wall. This is an approximate boundary condition and when it is implemented in the higher order scheme, oscillations and convergence problems are encountered and the following exact condition is derived for $\omega_{\text {wall }}$. By realizing that a $\left(1 / y^{2}\right)$ type singularity for the variable $\omega$ arises because of the balance between the dissipation term and the viscous diffusion term in the $\omega$ equation, the singularity is removed by rewriting the variable $\omega$ as

$$
\omega=\frac{C}{y_{n}^{2}} \omega_{1}
$$

where $y_{n}$ is the normal distance to the wall, $C$ is a constant and $\omega_{1}$ is the new variable which is now regular near the wall. When this is substituted into the $\omega$ equation the following equation is obtained for $\omega_{1}$, which is similar to the $\omega$ equation except for the source term. 


$$
\begin{aligned}
& \frac{\partial}{\partial t}\left(\rho \omega_{1}\right)+\frac{\partial}{\partial x_{j}}\left(\rho u_{j} \omega_{1}\right)=\gamma \rho \Omega^{2} y_{n}^{2} \operatorname{Re}+\frac{1}{\operatorname{Re}} \frac{\partial}{\partial x_{j}}\left[\left(\mu+\frac{\mu_{T}}{\sigma_{w}}\right) \frac{\partial \omega_{1}}{\partial x_{j}}\right] \\
& -\frac{\omega_{1}}{\operatorname{Re}} \frac{1}{y_{n}^{2}}\left[\beta \rho \omega_{1}-6\left(\mu+\frac{\mu_{T}}{\sigma_{w}}\right)\left(\frac{\partial y_{n}}{\partial x_{j}}\right)^{2}\right] \\
& \left(-\frac{2}{y_{n} \operatorname{Re}}\right)\left[\left(\mu+\frac{\mu_{T}}{\sigma_{w}}\right) \frac{\partial \omega_{1}}{\partial x_{j}} \frac{\partial y_{n}}{\partial x_{j}}+\frac{\partial}{\partial x_{j}}\left\{\left(\mu+\frac{\mu_{T}}{\sigma_{w}}\right) \omega_{1} \frac{\partial y_{n}}{\partial x_{j}}\right\}\right] \\
& +\frac{2}{y_{n}} u_{j} \rho \omega_{1} \frac{\partial y_{n}}{\partial x_{j}}+2\left(1-F_{1}\right) \sigma_{\omega 2} \frac{\rho}{\omega_{1}} \frac{\partial k}{\partial x_{j}} \operatorname{Re} y_{n}\left(\frac{\partial \omega_{1}}{\partial x_{j}} y_{n}-2 \frac{\partial y_{n}}{\partial x_{j}} \omega_{1}\right) .
\end{aligned}
$$

The value of $\omega_{1}$ at the wall becomes

$$
\omega_{1 \text { wall }}=\frac{6 \mu_{w}}{\beta \rho_{w}}\left(\frac{\partial y_{n}}{\partial x_{j}}\right)_{w}{ }_{w} .
$$

Here the variable $\omega$ is nondimensionalised by

$$
\omega=\frac{\omega^{*}}{\left(\frac{U_{0}^{2}}{v_{0}}\right)} \text { and } C=\frac{1}{\operatorname{Re}^{2}} .
$$

Hence the procedure is to use the $\omega_{l}$ equation for the first few points near the wall and switch to the $\omega$ equation away from the wall. In these computations, the $\omega_{1}$ equation is solved for the first ten points near the wall. Figure 3 shows the distribution of $k, \omega$ and $\omega_{1}$ near the wall and it is seen that this technique resolves the viscous layer smoothly even though $\omega$ is infinite at the wall.

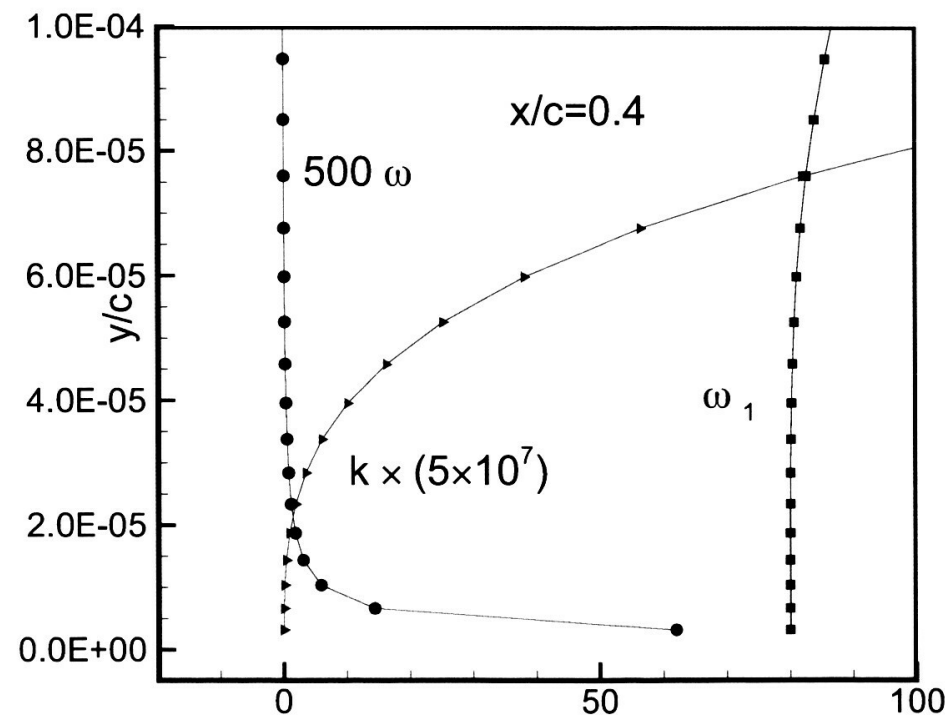

Figure 3: Variation of $k, \omega$ and $\omega_{1}$ near the wall at $x / c=0.4$. 


\section{Results}

Computations are performed for the baseline, the steady suction and the zero-mass oscillatory control cases. Figure 4 shows the comparison between the computed and the measured $u$-velocity profiles and the turbulent stress component $(u u)$ profiles at $x / c=-2.10$. This is the furthest upstream location where the measurements have been made. The length of the splitter plate in the computational model is selected to match the computed $u$-velocity profiles with the experiment at this station. It is seen that the computed $u$-velocity profile agrees well with the measured $u$-velocity profile, but the turbulent quantity is under predicted by a factor of two in the computations.
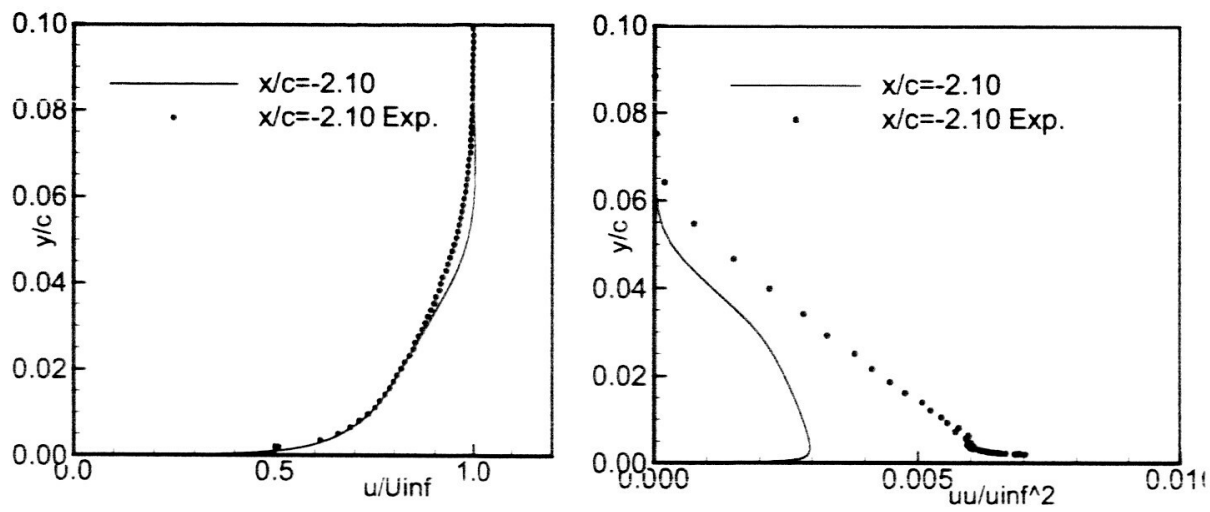

Figure 4. Comparison of velocity and turbulent quantity profiles at $x / c=-2.1$
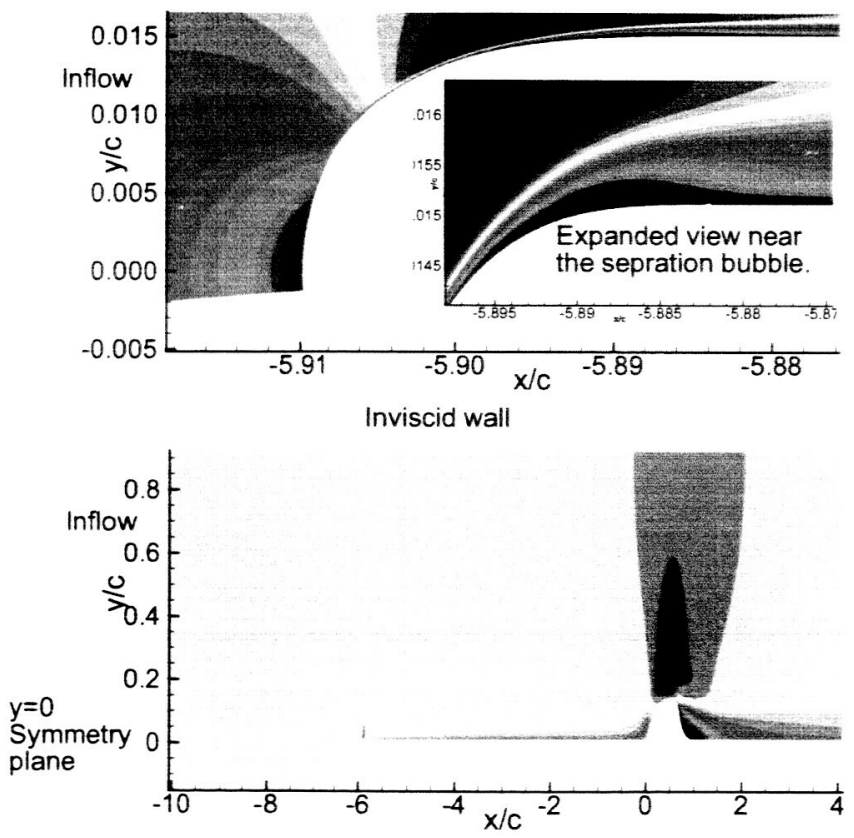

Figure 5: Contours of $u$ velocity near the leading edge and in the entire computational domain.

\section{A. Baseline and steady suction control cases}

Figure 5 shows the contours of the $u$ velocity near the leading edge region and for the entire computational domain. Near the leading edge region the flow separates and forms a small separation bubble. The smoothness of the solution in the overlap region shows the applicability of the interpolation 
technique in the overlap region in this higher order formulation. Figure 6 shows the computed and the measured $C p$ distribution for the baseline and the steady suction cases. Qualitatively the same phenomena are observed in both cases and quantitatively there are some discrepancies in the pressure distributions in the separation region.

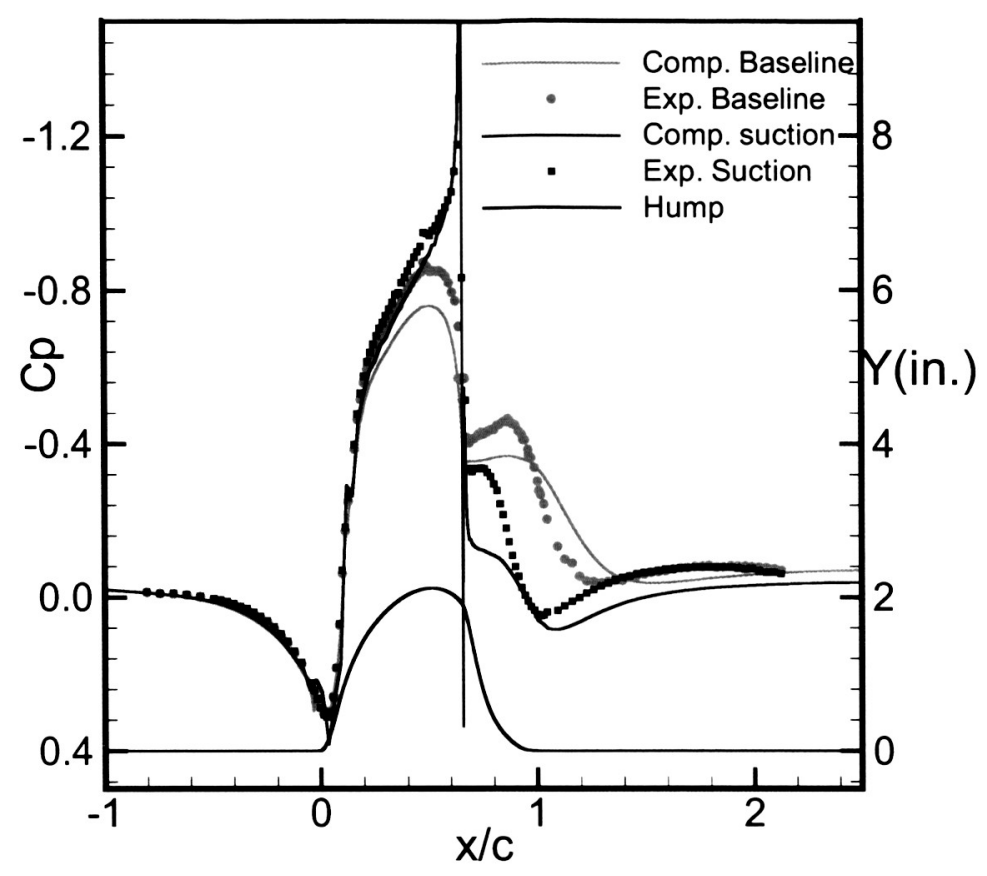

Figure 6. $C p$ distribution for the baseline and the steady suction control case.

The flow decelerates as it approaches the leading edge of the hump and then steeply accelerates up to $x / c=0.20$. In the baseline case, the acceleration is weakened after $x / c=0.20$ and the pressure coefficient peaks around $x / c=0.40$. Downstream the flow steeply decelerates up to $x / c=0.65$ and separates. The computed peak $C p$ is about $10 \%$ smaller than the experimental value. This is due to the blockage of the end plates. In the experiments, the non-dimensionalization is done using the measured values at $x / c=-2.10$ which is upstream of the end plates. Computational studies performed ${ }^{10}$, one using an altered top wall contour to approximately account for the end-plates blockage and another by solving the full three-dimensional problem including the end-plates, showed better agreement of the peak pressure with the experimental data and supported the conjecture that there exists a small blockage effect caused by the end plates. It is also observed that some differences exist in the $C p$ distribution between the computation and the experiment in the separation region and the separation bubble is longer in the computation. In the steady suction control case, the flow continues to accelerate up to the suction slot $x / c$ $=0.65$, and then steeply decelerates and separates at the slot. As expected the separation region becomes smaller compared to the baseline case and as in the no flow control case the separation region is longer in the computation compared to the experiment.

Figure 7 shows the streamline pattern for the computations and the experiments for the baseline and the steady suction cases. Table 2 . gives the separation point and the reattachment points obtained from the computations and the experiments for the base line and the suction cases. In the baseline case, the length of the separation bubble is about 0.42 in the experiments while it is 0.61 in the computations. When the flow is controlled using suction, the separation bubble lengths are 0.24 and 0.32 for the experimental and computational cases, respectively. 

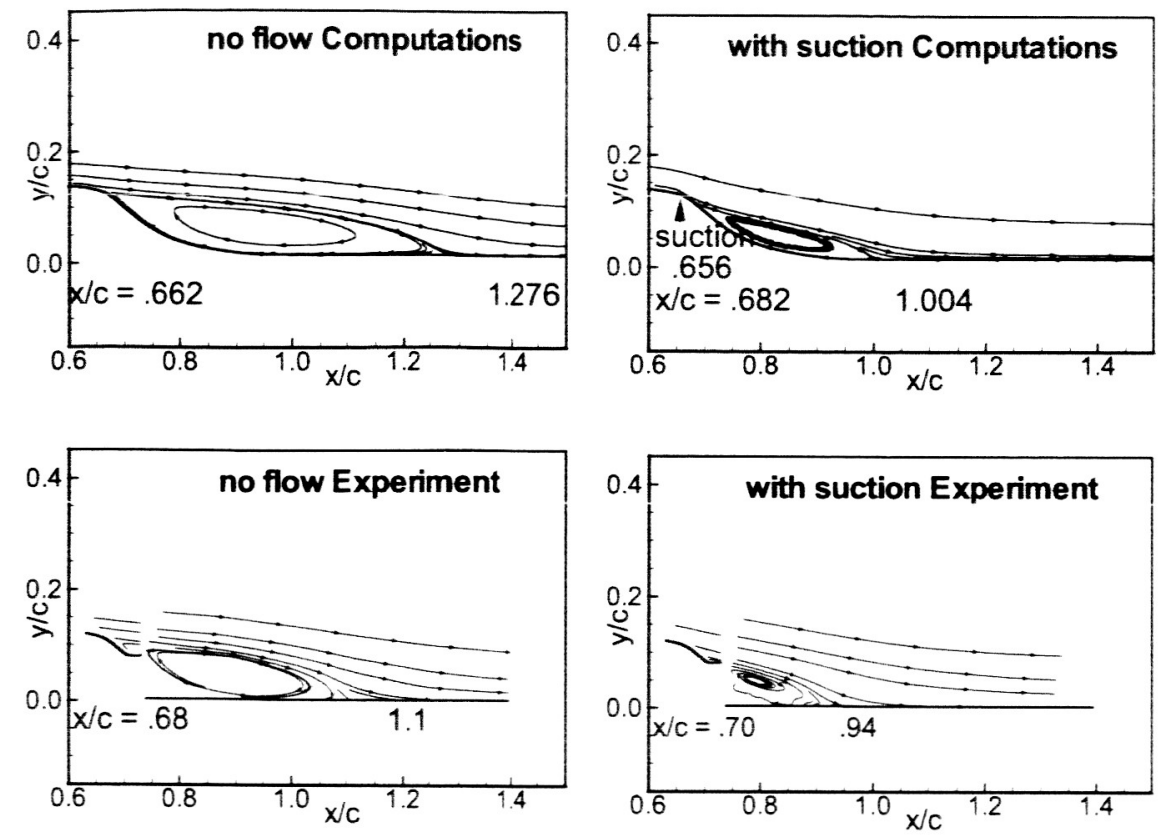

Figure 7. Comparison of separation region for the baseline and the steady suction control case between the computation and the experiment.

Figures 8 and 9 show the comparison between the computed and measured mean u-velocity profiles at $x / c=0.80,0.90,1.20$ and 1.30 for the baseline and the suction cases. Near the separation point, $x / c=0.80$ and 0.90 , the agreement between the computation and the experiment is good and the discrepancy increases near the reattachment region. This conforms to the earlier observation that the separation region is larger in the computation than in the experiment. Figures 10 and 11 show the comparison for the turbulent stress component $(u v)$. The agreement is not good for the turbulent quantities and the computations under predict the turbulent quantities by a factor of two to three. This points to the deficiencies in the turbulent model for the separated flows.
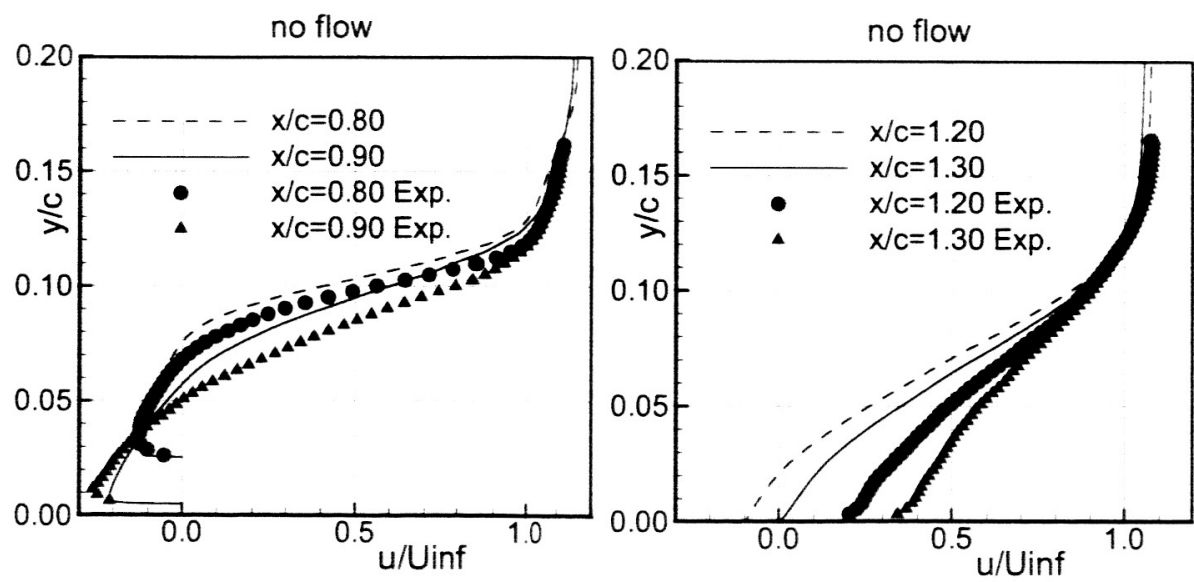

Figure 8. Comparison of mean velocity profiles for the baseline case. 

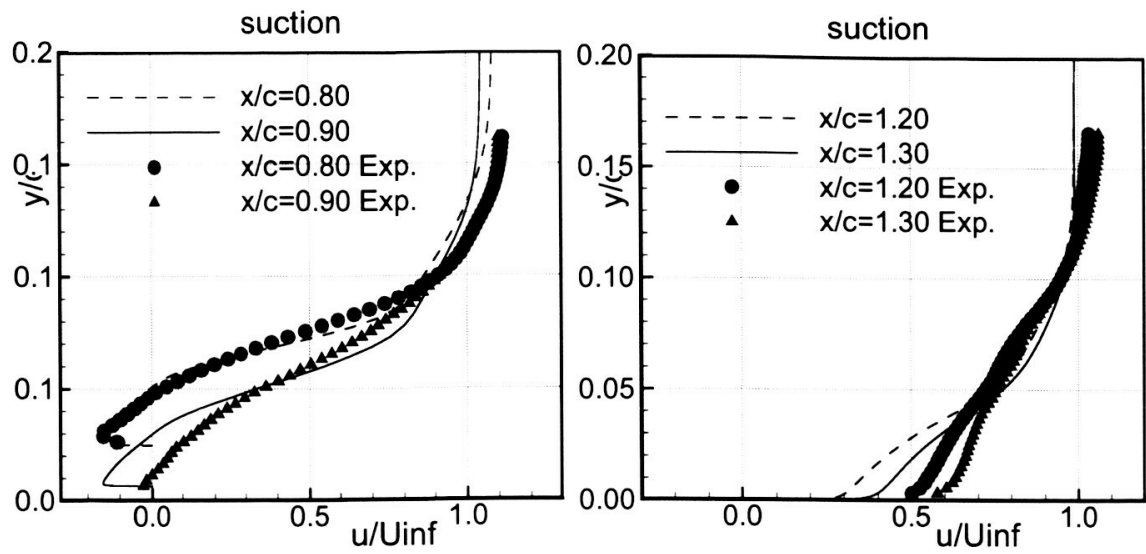

Figure 9. Comparison of mean velocity profiles for the suction case.
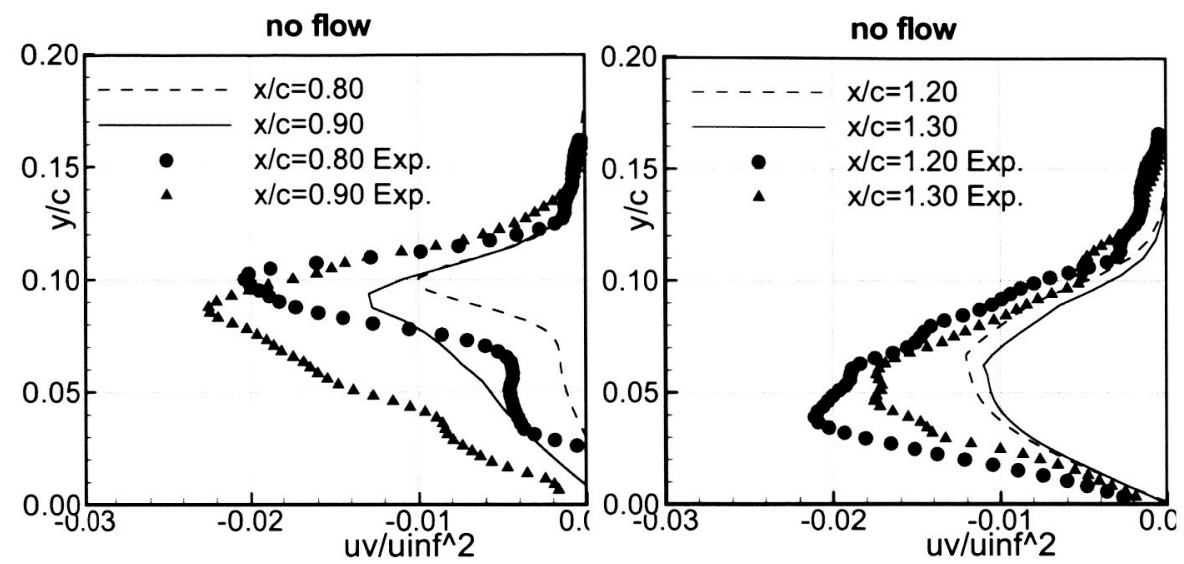

Figure 10. Comparison of turbulent stresses profiles for the baseline case.
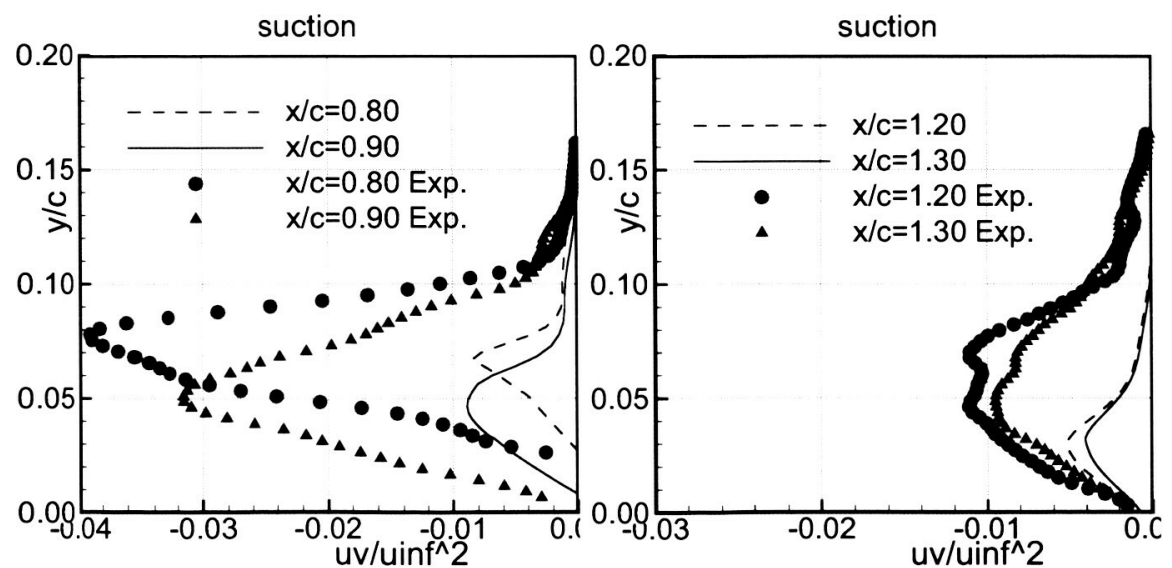

Figure 11. Comparison of turbulent stresses profiles for the suction case. 


\section{B. Oscillatory control}

After the baseline and steady suction control cases were computed time accurate computations were performed for the zero-mass flux blowing and suction control cases. Since this is an explicit code, the numerical instability limits the time step to a small value. It takes about $10^{6}$ time steps per cycle. The computations are continued until a periodic state in time is reached. Figure 12 shows the pressure as a function of phase angle (or time) for the last two cycles at a fixed location on the wall inside the separated region at $x / c=0.8$. It takes about 5-6 cycles before a periodic state is reached.

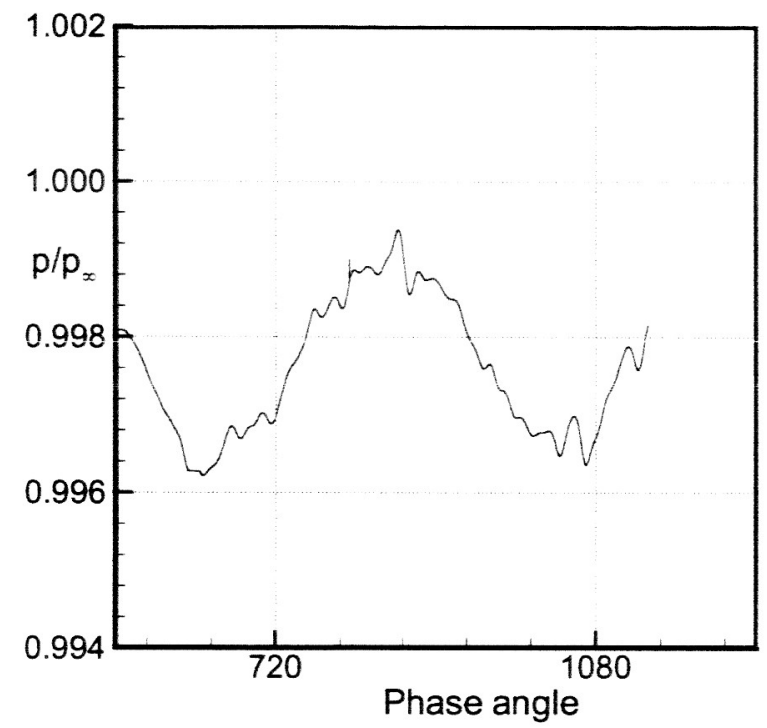

Figure 12. Pressure fluctuations as a function phase angle (or time) at a fixed point on the wall $x / c=0.8$.

Figure 13 shows the computed and the measured $C p$ distributions for the baseline and the oscillatory cases. The pressure distribution for the oscillatory case is the time averaged pressure for one cycle. Qualitatively the same phenomena are observed in both cases and quantitatively there are some discrepancies in the pressure distributions in the separation region. In the experiment, the pressure decreases immediately downstream of the slot, then increases up to the reattachment point. In the computations, it remains flat near the slot and then increases. As in other cases, the separation region is narrower in the experiment compared to the computations. 


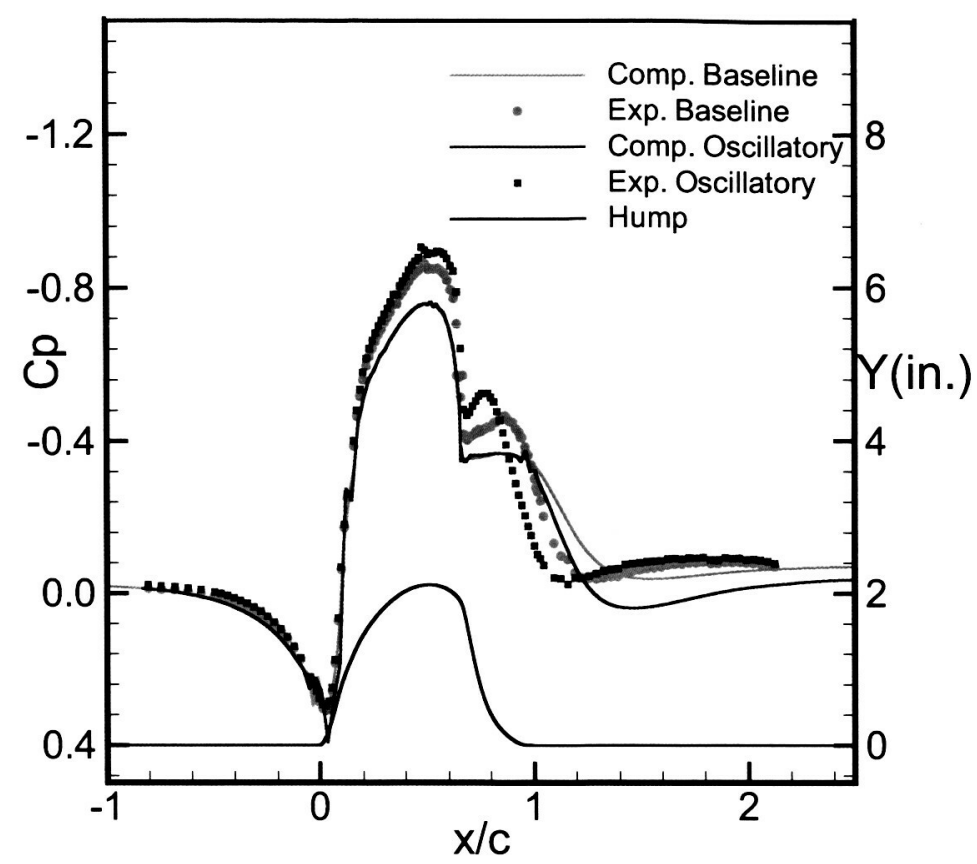

Figure 13. $C p$ distribution for the baseline and the oscillatory control case.

Figure 14 shows the mean streamline patterns in the separation zone obtained from the computation and the experiment. Clearly the separation region is smaller, about $20 \%$, in the experiment compared to the calculations. Table 2 summarizes the experimental and the computed separation and reattachment points for all three cases. The reattachment point for the unsteady control case is about $x / c=$ 1.21 in the simulation and about 0.98 in the experiment. In the experiment, the attachment point moved from $x / c=1.10$ for the baseline case to $x / c=0.98$ for the oscillatory control case and in the computations the attachment point moves from $x / c=1.276$ in the baseline case to $x / c=1.21$ in the control case. Even though the trend is in the right direction, the reduction in the separation is more in the experiment.
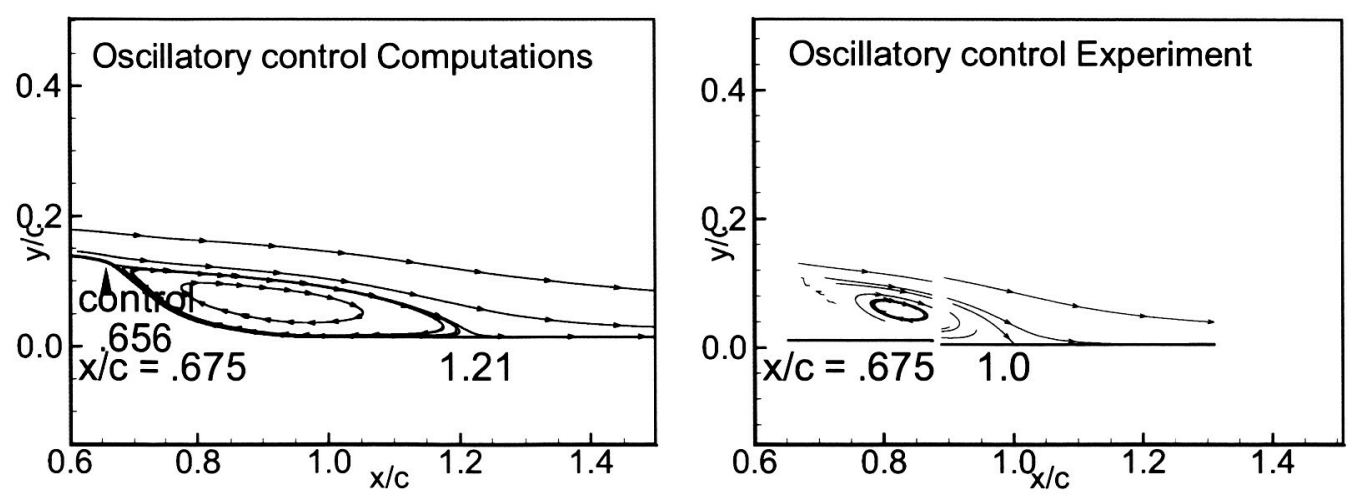

Figure 14. Comparison of separation region for the oscillatory control case. 
Table 2 Separation and reattachment locations

\begin{tabular}{|c|c|c|c|}
\hline \multicolumn{2}{|c|}{} & $\begin{array}{c}\text { Separation } \\
\text { Point } \boldsymbol{x} / \mathrm{c}\end{array}$ & $\begin{array}{c}\text { Reattachment } \\
\text { Point } \boldsymbol{x} / \boldsymbol{c}\end{array}$ \\
\hline \multirow{3}{*}{ Baseine } & Computation & 0.662 & 1.276 \\
\cline { 2 - 4 } & Experiment & 0.680 & 1.100 \\
\hline \multirow{3}{*}{ Oscillatory } & Computation & 0.682 & 1.004 \\
\cline { 2 - 4 } & Experiment & 0.700 & 0.940 \\
\cline { 2 - 4 } & Compütation & 0.675 & 1.21 \\
\hline & Experiment & 0.675 & 0.98 \\
\hline
\end{tabular}

Figures 15 compares the unsteady flow fields obtained from the computations and the experiments at different phases of the control cycle. The figure shows the contours of the $u$-velocity and the instantaneous streamline patterns at different phase angles $\phi=0,90,180$ and 270 degrees. Below each of the contour plots, the unsteady pressure coefficient, $\mathrm{Cp}-\mathrm{Cp}_{\text {mean }}$, at the corresponding phase angles are depicted. Here $\mathrm{Cp}_{\text {mean }}$ is the time averaged pressure coefficient shown in Fig. 13. The phase angles, $\phi=0$ and 180 degrees, correspond to the start of the blowing and suction phases of the control and $\phi=90$ and 270 degrees correspond to maximum blowing and suction phases. Qualitatively, the computed flow field structure and the unsteady pressure variations are similar to that are observed in the experiments. At the start of the cycle $\phi=0$, both in the experiments and computations, a small vortex forms near the slot and a large vortex precedes this small vortex downstream. Following the sequence, it is seen that these vortices become larger and move downstream with increasing phase angles. At the end of the blowing phase or the start of the suction of the suction phase $\phi=180$, there exist two co-rotating vortices in the separation zone. During the suction phase, the vortex closer to the slot becomes larger and the second vortex becomes weaker. The significant difference between the computation and the experiment is the appearance of a large vortex in the computation near the reattachment region. This vortex persists as an elongated vortex during the blowing phase. This implies that the suction phase is not as effective in the computations as in the experiments in pulling the separation region up towards the slot. It may be that there is not sufficient turbulent mixing in the reattachment region to bring the high momentum fluid towards the wall. This may be the reason for over predicting the separation region in the computations.

\section{Conclusions}

Turbulent separated flow over a two-dimensional hump is computed by solving the RANS equations with $k$ - $\omega$ (SST) turbulence model using a fifth-order accurate WENO method for the baseline, steady suction and zero mass oscillatory control cases. Overlapping grids are used to compute the leading edge region. The singularity in the $\omega$ equation near the wall is removed by solving the $\omega$ equation in the transformed plane and this also removes the ambiguity in applying the wall boundary condition for $\omega$ that exists now in practice.

Qualitatively the computed pressure distributions exhibit the same behavior as those were observed in the experiments. The computed separation regions are much longer in the computations than in the experiments. Computations verified that steady suction could be used to significantly reduce the separated region in a flow. However, the computations predict a small reduction in separated region compared to the experiment. The predicted turbulent quantities are two to three times smaller than that are 
measured and it points towards the deficiencies in the existing turbulent models when they are applied to strong separated steady and unsteady flows.

Time accurate computations of the unsteady zero mass oscillatory control case revealed similar flow field structures and unsteady pressure variations in the separated region. However, the computations did not show the expected large percentage reduction in the separated region compared to the experiment. In the computations an additional vortex appears near the reattachment region and in the experiment this was not observed. This may be due to less turbulent mixing in this region. 
Comp. Phase angle 0
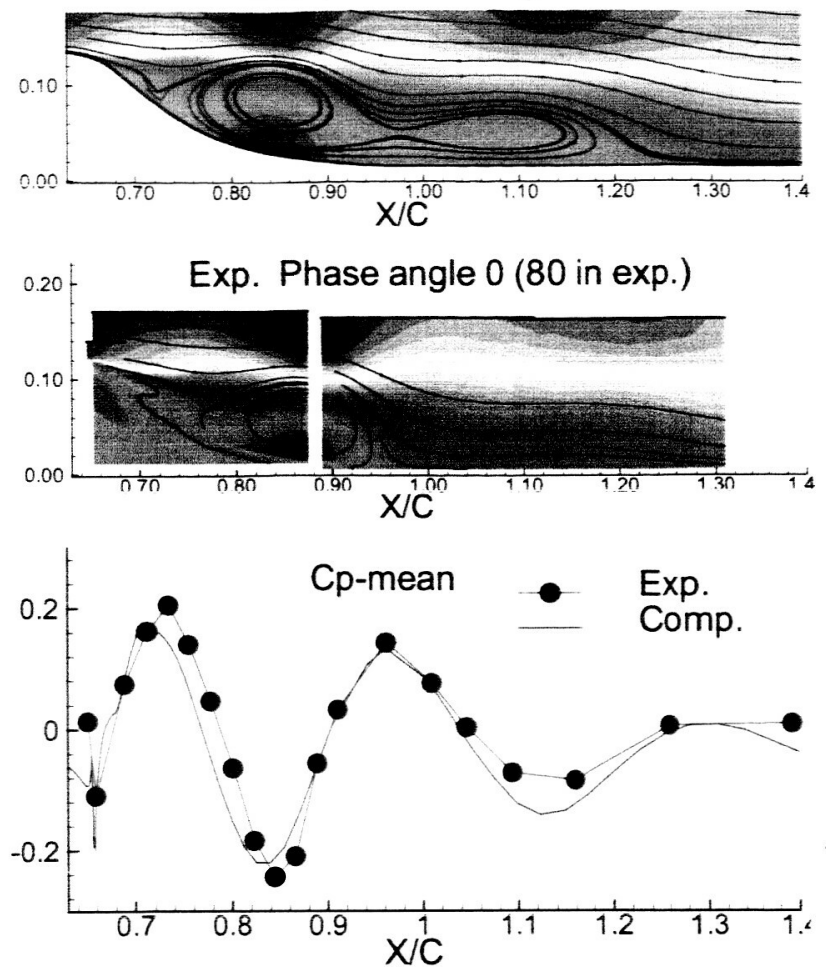

Comp. Phase angle 180
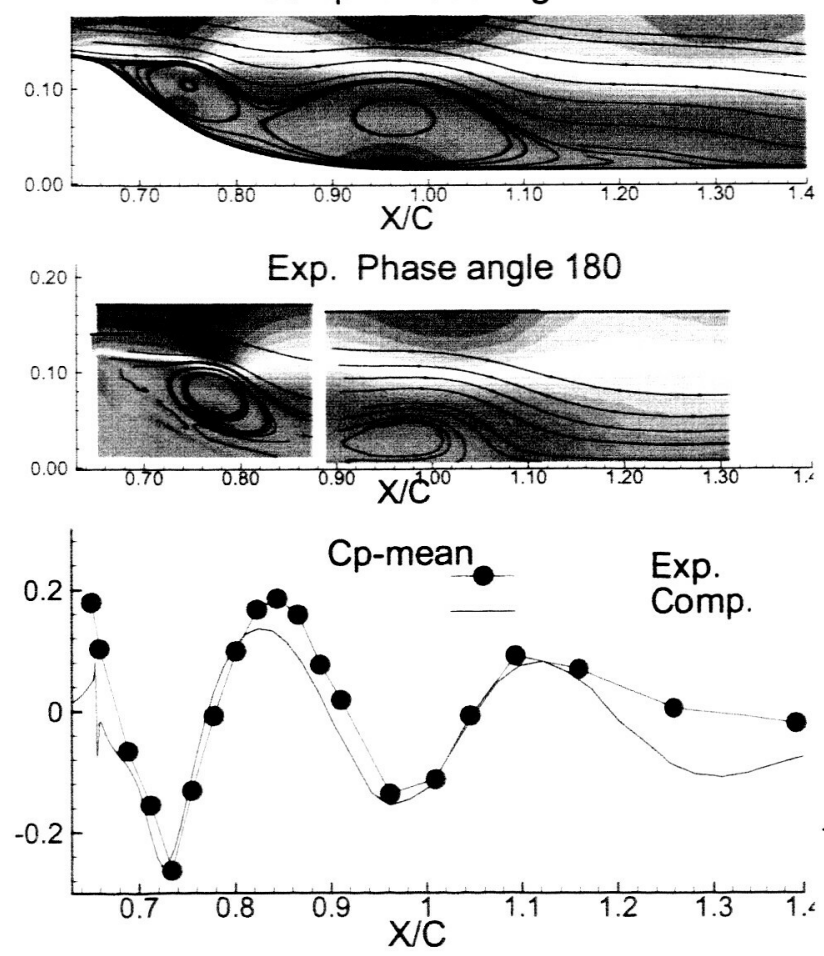

Comp. Phase angle 90 Blowing maximum
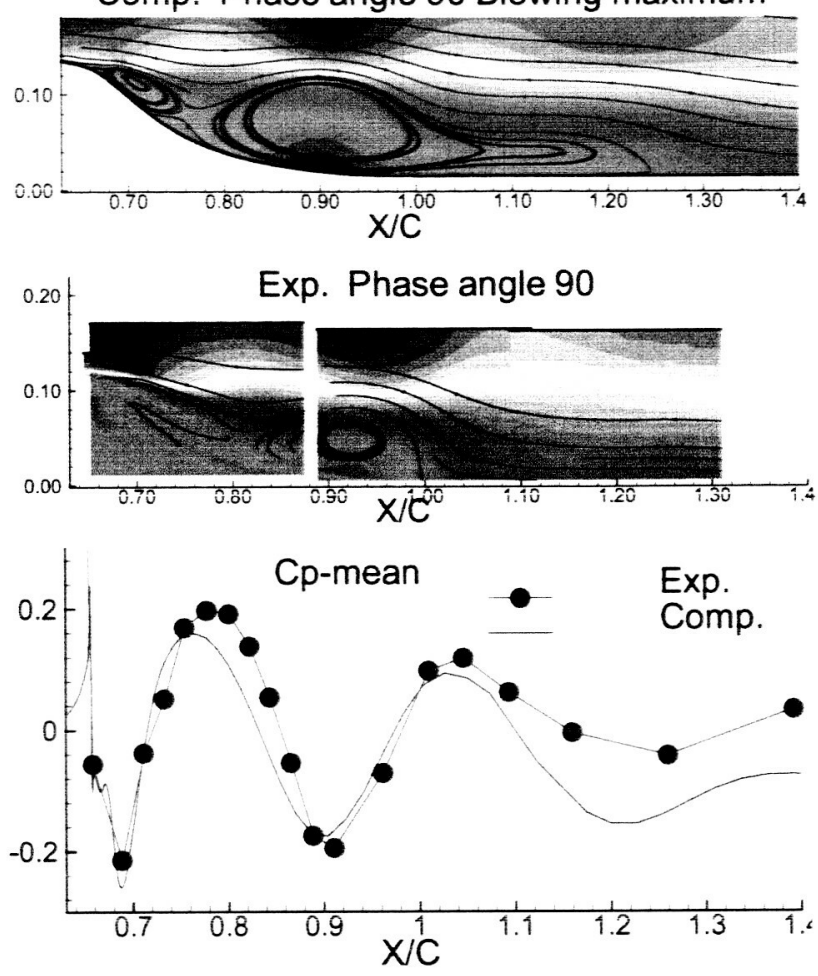

Comp. Phase angle 270 Suction maximum
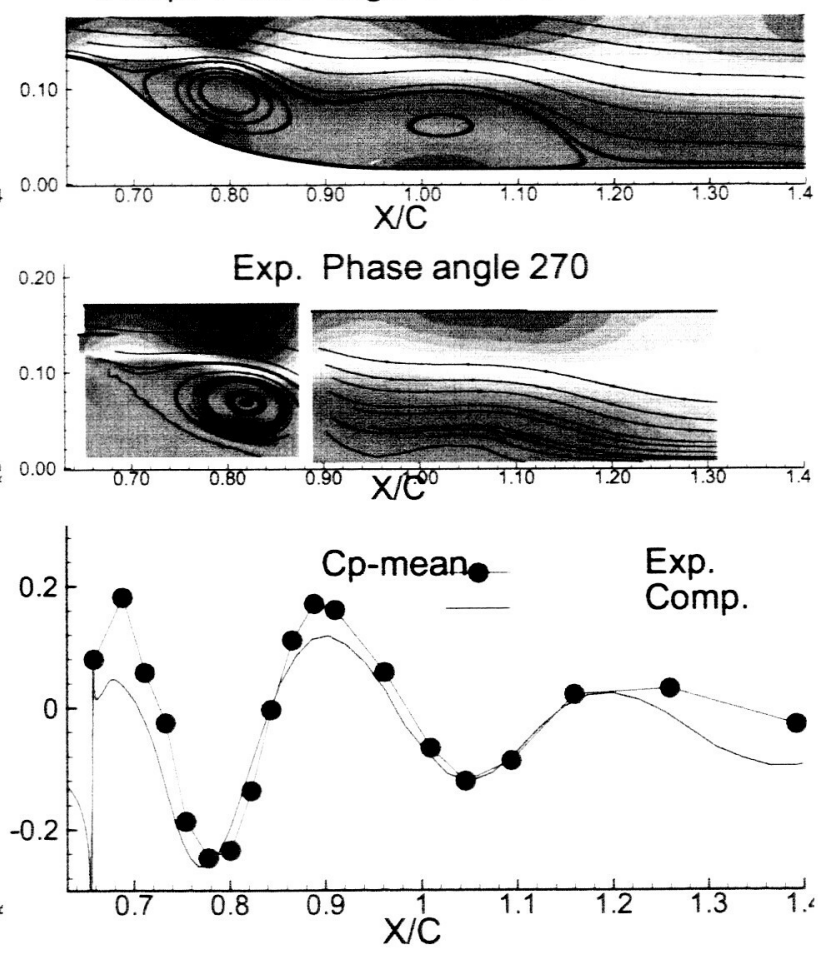

Figure 15. Comparison of the flow field and the oscillatory pressure in the separation region for the oscillatory control. 


\section{References}

1. Carpenter, M. H., Singer, B. A., Yamaleev, N., Vatsa, V. N., Viken, S. A., and Atkins, H. L., "The Current Status of Unsteady CFD Approaches for Aerodynamic Flow Control," AIAA Paper 2002-3346, 2002.

2. Lele, S. K., "Compact Finite Difference Schemes with Spectral-Like Resolution," Journal of Computational Physics, 103 (1992), 16-42.

3. Harten, A., Engquist, S., Osher, S., and Chakravarthy, S., "Uniformly High Order Essentially Non-Oscillatory Schemes III," Journal of Computational Physics, 71 91987), 231-303.

4. Visbal, M. R. and Gaitonde, D. V., "High-Order Accurate Methods for Unsteady Vortical Flows on Curvilinear Meshes," AIAA Paper 1998-0131, 1998.

5. Gaitonde, D. V. and Visbal, M. R., "Further Development of a Navier-Stokes Solution Procedure Based on Higher-Order Formulas," AIAA Paper 1999-0557, 1999.

6. Visbal, M. R. and Gaitonde, D. V., "High-Order Accurate Methods for Unsteady Vortical Flows on Curvilinear Meshes," AIAA Paper 1998-0131, 1998.

7. Balakumar, P., Zhao, H., and Atkins, H. L., "Stability of Hypersonic Boundary-Layers over a Compression Corner," AIAA Paper 2002-2848, 2002.

8. Balakumar, P., "Transition in a Supersonic Boundary layer due to Roughness and Acoustic Disturbances," AIAA Paper 2003-3589, 2003.

9. Imamoglu, B., and Balakumar, P., "Computations of Shock Induced Noise in Imperfectly Expanded Supersonic Jets," AIAA 2002-2527, 2002.

10. Rumsey, C. L., Gatski, T. B., Sellers, W. L., Vatsa, V. N., and Viken, S. A., "Summary of the 2004 CFD Validation Workshop on Synthetic jets and Turbulent Separation Control," AIAA Paper 2004-2217, 2004.

11. Greenblatt, D., Paschal, K. B., Schaeffler, N. W., Washburn, A. E., Harris, J., and Yao, C. S., "A Separation Control CFD Validation Test Case. Part 1: Baseline and Steady Suction," AIAA Paper 2004-2220, 2004.

12. Seifert, A. and Pack, L. G., "Active Flow Separation Control on Wall-Mounted Hump at High Reynolds Numbers," AIAA Journal, Vol. 40, No. 7, July 2002.

13. Shu, Chi-Wang, "Essentially Non-Oscillatory and Weighted Essentially Non-Oscillatory Schemes for Hyperbolic Conservation Laws," NASA/CR-97-206253 and ICASE Report N0. 9765, 1997.

14. Atkins, H. L., "High-Order ENO Methods for the Unsteady Compressible Navier-Stokes Equations," AIAA Paper 91-1557, 1991.

15. Menter, F. R., "Improved two-Equation k- $\omega$ Turbulence Models for Aerodynamic Flows," NASA Technical Memorandum, N0. 103975, 1992.

16. Menter, F. R., “Zonal Two Equation k- $\omega$ Turbulence Models for Aerodynamic Flows," AIAA Paper 93-2906, 1993.

17. Menter, F. R. and Rumsey, C. L., "Assessment of Two-equation Turbulence Models for transonic Flows," AIAA Paper 94-2343, 1994.

18. Krist S. L., Biedron R. T., and Rumsey C. L., "CFL3D User's Manual (Version 5)," NASA TM1998-208444, June 1998.

19. Sesterhenn, J., "A Characteristic-type Formulation of the Navier-Stokes Equations for High Order Upwind Schemes," Computers \& Fluids, 30, pp. 37-67, 2001. 